\title{
Pseudobulbar Affect Following Trans-Cerebellar Resection of A Hemorrhagic Pontine Cavernous Malformation: A Case Report
}

\author{
Samer S Hoz, MD, FRCS ${ }^{1}$, Zahraa F Al-Sharshahi, MD ${ }^{1 *}$, Gheyath Algawwam, MD ${ }^{2}$ and Anshit Goyal, MD \\ ${ }^{1}$ Department of Neurosurgery, Neurosurgery Teaching Hospital, Baghdad, Iraq \\ ${ }^{2}$ Neurologist, Assistant Professor in Neurology, Department of Neurology, Baghdad University, College of \\ Medicine, Baghdad, Iraq \\ ${ }^{3}$ Department of Neurologic Surgery, Mayo Clinic, Minnesota, USA
}

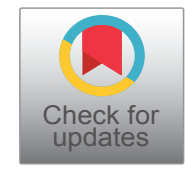

*Corresponding author: Zahraa FAl-Sharshahi, Department of Neurosurgery, Neurosurgery Teaching Hospital, Baghdad, Iraq, Tel: 0096478216219848

\begin{abstract}
Background: The emergence of psychiatric symptoms purely as a sequela of brain lesions is a rare occurrence. The exact neural circuits governing such manifestations are poorly understood; their early recognition is, nonetheless, of pivotal importance to patient management.

Case presentation: Herein, we report a case of a 23-year-old male patient who presented with a new-onset pseudobulbar affect following trans-cerebellar resection of a hemorrhagic pontine cavernous malformation. Following a one-month course of $5 \mathrm{mg}$ Olanzapine, the patient's affect improved, although a residual disinhibited behavior was stil evident. Neurologically, the patient achieved near-complete recovery.

Conclusion: The link between brain lesions and psychiatric disorders is poorly established, yet, it is an important differential to consider in post-operative psychiatric disturbances.
\end{abstract}

\section{Keywords}

Cavernous malformation, Pseudobulbar affect, Pontine cavernoma

\section{Abbreviations}

CM: Cavernous Malformation; CT: Computerized Tomography; MRI: Magnetic Resonance Imaging; DVA: Developmental Venous Anomaly

\section{Background}

Psychiatric manifestations are rarely encountered following surgery for infratentorial lesions. To date, four types of psychiatric and cognitive abnormalities associated with brainstem lesions have been reported in the literature; namely, peduncular hallucinosis [1], psychosis [2,3], steroid-induced psychosis [4], and pseudobulbar affect [5]. The exact pathophysiology underlying these presentations is not yet understood. However, some reports have suggested that hemorrhagic or infectious lesions of the brainstem and the cerebellum may affect their supratentorial connections to the thalamus and the limbic system, producing these psychiatric features [6]. Here, we present the first report of pseudobulbar affect following surgical resection of a brainstem lesion.

\section{Case Presentation}

A 23-year-old male presented to the emergency department with acute onset drowsiness, left-sided ataxia, and nystagmus. He had a known cavernoma compressing the fourth ventricle with two previous episodes of hemorrhage.

The MRI scan confirmed the diagnosis of a large dorsomedial hemorrhagic pontine cavernous malformation (CM). The $\mathrm{CM}$ was associated with a significant developmental venous anomaly (DVA) that passed medially then superiorly to be drained before draining into the Galenic venous system (Figure 1A). Based on the acute presentation, location, and size of the hematoma along with the history of recurrent

Citation: Hoz SS, Al-Sharshahi ZF, Algawwam G, Goyal A (2021) Pseudobulbar Affect Following TransCerebellar Resection of A Hemorrhagic Pontine Cavernous Malformation: A Case Report. Neurosurg Cases Rev 4:082. doi.org/10.23937/2643-4474/1710082

Accepted: October 07, 2021; Published: October 09, 2021

Copyright: (C) $2021 \mathrm{Hoz}$ SS, et al. This is an open-access article distributed under the terms of the Creative Commons Attribution License, which permits unrestricted use, distribution, and reproduction in any medium, provided the original author and source are credited. 


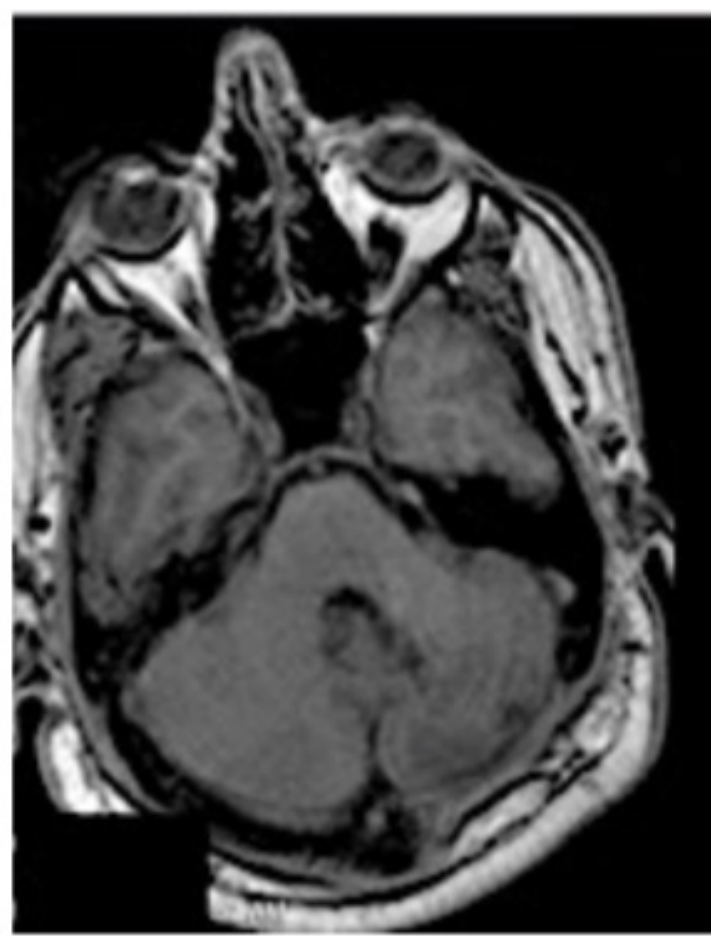

A

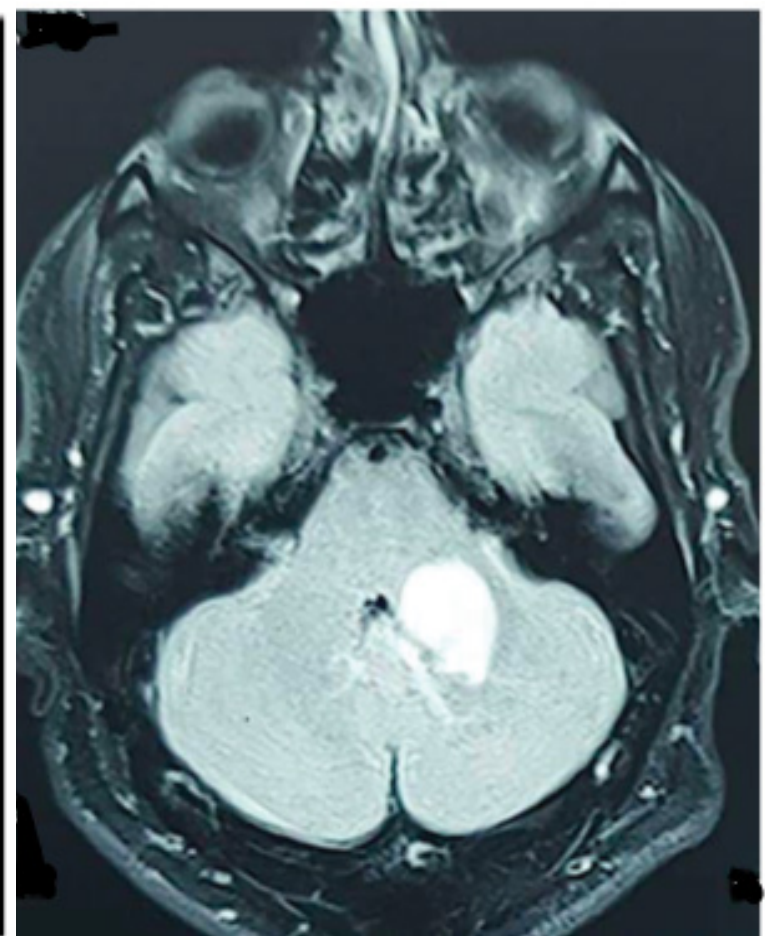

$\mathrm{B}$

Figure 1: Hemorrhagic pontine cavernous malformation; A) Pre-operative axial FLAIR MRI, showing the dorso-medial hemorrhagic pontine cavernous malformation compressing the $4^{\text {th }}$ ventricle without causing hydrocephalus; B) Postoperative axial T-weighted MRI, showing the complete resection of the cavernous malformation using a posterior transcerebellar approach.

hemorrhages, a decision was made to evacuate the hematoma urgently.

With the patient in a prone position, a left transcerebellar approach was opted for through the left suboccipital craniectomy. The hematoma was evacuated, and the CM was completely resected using a microsurgical technique. The associated DVA was preserved in order to avoid any unwanted events. After gentle transgression of the cerebellar cortex, the CM was approached using the horizontal cerebellar crack as a narrow corridor to access the lesion from the nearest cortical point. The CM was then evacuated by internal debunking until a complete microsurgical resection was achieved.

Two hours postoperatively, the patient was alert, oriented, and appropriately following commands in all four limbs. However, he was disinhibited, screaming, laughing with attacks of combativeness, and using inappropriate words with the medical staff. An urgent CT scan of the brain did not show any hematoma, hydrocephalus, or frontal pneumocephalus (Figure 2). A comprehensive metabolic panel revealed no electrolyte derangement, and a review of the patient's charts ruled out peri-operative steroids as a potential culprit. Electroencephalography provided no significant findings. Intravenous phenobarbital and analgesia were administered over the next two days.

A psychiatric consultation confirmed the diagnosis of pseudobulbar affect, and the patient was started on Olanzapine (5 $\mathrm{mg}$ twice daily tablets). The patient became non-combative but still exhibited disinhibition and inappropriate affect. Over the ensuing ten days, his condition gradually improved, and he was discharged with significant neurological improvement.

During his two-week follow-up visit, the family reported an improvement in the patient's attitude and mood with residual disinhibition. He was therefore continued on Olanzapine for another two weeks. Two weeks later, the patient was shown to have an appropriate affect without disinhibition, and Olanzapine was, hence, discontinued. His three-month followup MRI scan revealed the complete evacuation of the $\mathrm{CM}$ with the preservation of the adjacent DVA. Except for a mild persistent left-sided ataxia, the patient's neurological examination was essentially unremarkable without any neuropsychiatric findings (Figure 1B).

\section{Discussion}

Pseudobulbar affect is a brainstem-related psychiatric disorder characterized by disinhibited behavior along with inappropriate affect and pathological laughter and crying [5]. Pseudobulbar affect has not been previously reported as a complication of brain stem surgery. However, a spectrum of rare psychiatric disorders has been reported as a manifestation of the brainstem and cerebellar lesions [7,8]. In rare instances, brainstem lesions may also be associated with peduncular 

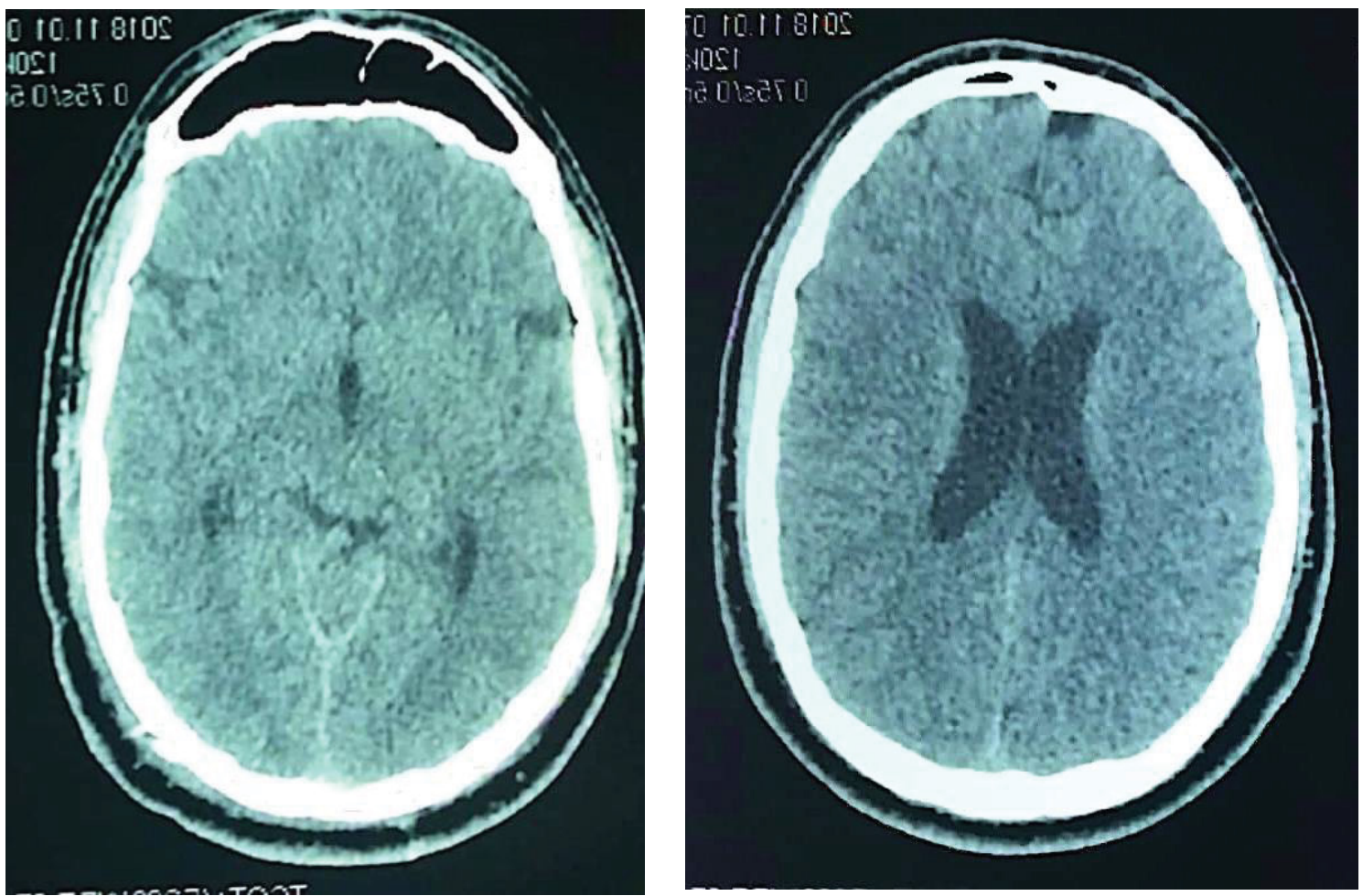

Figure 2: Post-operative Axial CT scan showing normal frontal lobe.

hallucinosis. The cornerstone diagnostic feature for peduncular hallucinosis is the presence of visual or auditory hallucinations $[7,8]$.

Although Couse, et al. [9] have reported peduncular hallucinosis to be an atypical presenting feature of cavernous malformations in the pons, this is not applicable to our case as the patient was psychiatric symptom-free pre-operatively. Frank psychosis has also been reported as an extremely rare feature of midbrain lesions often presenting with odd thoughts and hyperreligiosity [2]. Long term steroid use has also been linked with "steroid psychosis," which manifests as depression, emotional lability, and sleep disturbances [4]. Steroid psychosis is not a well-defined term and has been subject to many arguments in the literature [10]. Our patient did not receive a long course of steroids, excluding the possibility of steroid psychosis.

The neuronal circuits responsible for the psychiatric manifestations of some infratentorial lesions are not well understood. Some reports have suggested that vasospasm in the brainstem perforators and the resultant ischemia of the dorsal raphe nucleus may cause psychiatric presentations after brainstem injury [6]. A number of experimental studies have also connected the cerebellum and cerebro-cerebellar pathways to additional functions related to memory, behavior, and mood. These connections may provide an explanation for some of the psychiatric disorders related to cerebellar lesions in the future [11-14].

Traditionally, surgical approaches for CM proximal to the lateral recess included the trans-lateral recess telovelar approach, the trans cerebellomedullary fissure approach, pre-sigmoid approach, and, rarely, the anterior endoscopic approach [15]. The presence of a medially located large developmental venous anomaly along with the $\mathrm{CM}$ may render the common approaches not feasible. Ultimately, the surgical strategy for infratentorial CM involves two critical goals: First, the CM should be totally excised, while preserving the normal, surrounding parenchyma, to reduce the risk of recurrent hemorrhage. Second, extreme care should be taken to preserve the associated developmental venous anomaly to prevent an ischemic injury to the surrounding normal parenchyma.

To date, the optimal treatment for pseudobulbar affect is debatable, with a variety of typical and atypical antipsychotic drug options being available, including Olanzapine, Clozapine, Valproate, Fluoxetine, and Risperidone $[6,15,16]$.

In conclusion, posterior fossa surgery is a rare cause of psychiatric symptoms, such as pseudobulbar affect. By being cognizant about the possibility of such connections, surgeons may become more adept at recognizing and treating these symptoms. Nevertheless, the exact neuronal pathways governing these links remain to be elucidated.

\section{Learning Points}

- Pseudobulbar affect is a rare complication of surgeryfor infratentorial cavernous malformation.

- Injury to the brainstem or cerebellum may result in various psychiatric manifestations. 
- Exclusion of supratentorial causes should be considered first when psychiatric complications are encountered following infratentorial surgery.

\section{Declarations}

\section{Ethics approval and consent to participate}

None-required.

\section{Consent for publication}

None-required.

\section{Availability of data and materials}

Yes.

\section{Competing interests}

Nothing to declare.

\section{Funding}

None-received.

\section{Acknowledgements}

None.

\section{Author Contributions}

S.S.H: Case identification and manuscript review; Z.A: Literature review, drafting of manuscript; G.A.: Reviewing and revising original draft; A.G.: Reviewing and revising original draft.

\section{References}

1. Dogan VB, Dirican A, Koksal A, Baybas S (2013) A case of peduncular hallucinosis presenting as a primary psychiatric disorder. Ann Indian Acad Neurol 16: 684-686.

2. Andrews JP, Taylor J, Saunders D, Qayyum Z (2016) Peduncular psychosis. BMJ Case Reports.

3. Bielawski M, Bondurant $H$ (2015) Psychosis following a stroke to the cerebellum and midbrain: A case report. Cerebellum \& Ataxias.
4. Ross DA, Cetas JS (2012) Steroid psychosis: A review for neurosurgeons. J Neurooncol 109: 439-447.

5. Miller A, Pratt H, Schiffer RB (2011) Pseudobulbar affect: The spectrum of clinical presentations, etiologies and treatments. Expert Rev Neurother 11: 1077-1088.

6. Mocellin R, Walterfang M, Velakoulis D (2006) Neuropsychiatry of complex visual hallucinations. Aust N Z J Psychiatry 40: 742-751.

7. Benke T (2006) Peduncular hallucinosis: A syndrome of impaired reality monitoring. J Neurol 253: 1561-1571.

8. Feinberg WM, Rapcsak SZ (1989) Peduncular hallucinosis' following paramedian thalamic infarction. Neurology 39: 1535-1536.

9. Couse M, Wojtanowicz T, Comeau S, Bota R (2018) Peduncular hallucinosis associated with a pontine cavernoma. Ment IIIn 10: 14-15.

10. Decramer T, Demaerel P, Lemmens R (2015) A frontal syndrome due to a brainstem lesion. Acta Neurol Belg 115: 683-684.

11. Andreasen NC, Pierson R (2008) The role of the cerebellum in schizophrenia. Biol Psychiatry 64: 81-88.

12. Manto M, Marien P (2015) Schmahmann's syndrome identification of the third cornerstone of clinical ataxiology. Cerebellum Ataxias 2: 2.

13. Schmahmann J, Sherman J (1998) The cerebellar cognitive affective syndrome. Brain 121: 561-579.

14. Shakiba $A$ (2014) The role of the cerebellum in neurobiology of psychiatric disorders. Neurol Clin 32: 1105-1115.

15. Almeida J, Serrao EM, Almeida AT, Afonso JG (2011) Effective treatment with clozapine and valproate for refractory schizophrenia-like psychosis after cerebellar hemorrhage. Clin Neuropharmacol 34: 131-132.

16. Duggal HS (2005) Cognitive affective psychosis syndrome in a patient with sporadic olivopontocerebellar atrophy. J Neuropsychiatry Clin Neurosci 17: 260-262. 